\title{
Substrate utilization and maturation of cumulus cell-enclosed mouse oocytes: evidence that pyruvate oxidation does not mediate meiotic induction
}

\author{
S. M. Downs ${ }^{1 *}$, F. D. Houghton ${ }^{2}$, P. G. Humpherson ${ }^{2}$ and \\ H. J. Leese ${ }^{2}$ \\ ${ }^{1}$ Department of Biology, Marquette University, Milwankee, WI 53201-1881, USA; and ${ }^{2}$ Department of \\ Biology, University of York, York YO1 5DD, UK
}

\begin{abstract}
This study was performed to address the possible role of pyruvate in meiotic induction in mouse oocytes. Cumulus cell-enclosed oocytes from primed, immature mice were cultured in $7.5 \mu \mathrm{l}$ microdrops under oil for 9 or $18 \mathrm{~h}$ in medium containing $4 \mathrm{mmol}$ hypoxanthine $\mathrm{I}^{-1}$ plus $0.23 \mathrm{mmol}$ pyruvate $\mathrm{I}^{-1}, 1 \mathrm{mmol}$ pyruvate $\mathrm{l}^{-1}$, or $1 \mathrm{mmol}$ pyruvate $\mathrm{l}^{-1}$ plus $5.5 \mathrm{mmol}$ glucose $\mathrm{l}^{-1}$. When compared with cultures containing $0.23 \mathrm{mmol}_{\text {pyruvate }} \mathrm{l}^{-1}$, $1 \mathrm{mmol}$ pyruvate $1^{-1}$ induced germinal vesicle breakdown, and this was preceded by an increase in pyruvate utilization. Addition of glucose prevented both the increase in pyruvate consumption and the meiotic induction. When different combinations of pyruvate and glucose were tested on oocyte maturation in microdrop cultures, a high concentration of pyruvate or glucose alone was stimulatory to maturation. Addition of the complementary energy substrate prevented the induction of germinal vesicle breakdown and reduced the amount of substrate consumption. During spontaneous maturation in vitro, oocyte-cumulus cell complexes consumed glucose for the first $3 \mathrm{~h}$; however, during the second $3 \mathrm{~h}$ period, which followed germinal vesicle breakdown, glucose consumption decreased and net pyruvate utilization was initiated. Treatment of hypoxanthine-arrested oocytes with dichloroacetate, an activator of pyruvate dehydrogenase, stimulated pyruvate consumption but had no effect on germinal vesicle breakdown. Although FSH stimulates meiotic resumption, no changes in pyruvate consumption were observed in response to this gonadotrophin. Measurement of oxygen consumption by hypoxanthine-treated complexes revealed no effect of high concentrations of pyruvate on respiration, and FSH treatment resulted in a suppression of oxygen utilization. These data indicate that, in mouse oocyte-cumulus cell complexes, pyruvate and glucose can each modulate metabolism of the other substrate, and this can significantly influence meiotic maturation of the oocyte. In addition, augmentation of pyruvate oxidation does not appear to play a mediating role in meiotic induction triggered by energy substrate manipulation or gonadotrophin treatment.
\end{abstract}

\section{Introduction}

When mammalian oocytes that are fully grown and at the germinal vesicle stage are removed from the ovary and placed in a suitable medium, resumption of meiotic maturation occurs spontaneously (Pincus and Enzmann, 1935; Edwards, 1965). However, oocyte maturation in vitro can be affected by the presence or absence of accompanying follicle cells or by modifications of the culture medium. For example, glucose as a sole energy source can support the meiotic maturation of cumulus cell-enciosed, but not denuded, mouse oocytes (Biggers et al., 1967). This is because oocytes have a limited ability to metabolize glucose but cumulus cells can convert it to

*Correspondence.

Received 29 August 1990. pyruvate, which can be utilized subsequently by the oocyte (Biggers et al., 1967; Donahue and Stern, 1968; Eppig, 1976; Leese and Barton, 1985). Thus, cumulus cell-enclosed rodent oocytes will undergo maturation in glucose alone, whereas denuded oocytes require simpler carboxylic acids such as pyruvate or oxaloacetate (Biggers et al., 1967; Zeilmaker and Verhamme, 1974).

Changes in medium supplementation can also influence meiotic arrest and hormone induction of maturation. If meiotic inhibitors such as agents that increase cAMP concentration or purines are added to the culture medium, spontaneous germinal vesicle breakdown (GVBD) is prevented (Eppig and Downs, 1984; Schultz, 1991). These meiotic blocks can be eliminated by manipulating energy substrate (Downs and Mastropolo, 1994; Downs, 1995) or amino acid (Downs and Mastropolo, in press) 
supplementation, or by including stimulatory ligands such as FSH or epidermal growth factor in the culture medium (Dekel and Beers, 1978; Downs et al., 1988). Elimination of glucose and an increase in the pyruvate concentration can remove the maturation-blocking action of dibutyryl cAMP or hypoxanthine (Fagbohun and Downs, 1992; Downs and Mastropolo, 1994). Glycolytic metabolism of glucose helps generate ATP that apparently contributes to the meiosis-suppressing action of these inhibitory agents (Downs and Mastropolo, 1994; Downs, 1995). Nevertheless, the meiosis-inducing action of FSH on inhibitor-treated oocytes is dependent on glucose (Fagbohun and Downs, 1992; Downs and Mastropolo, 1994). A recent study has shown that treating mouse oocyte-cumulus cell complexes with FSH stimulates glucose utilization; this is mediated, at least in part, by the augmentation of hexokinase activity and is sensitive to protein synthesis inhibition (Downs et al., 1996). This glucose consumption is accompanied by increased pyruvate production.

Because pyruvate can feed into the tricarboxylic acid (TCA) cycle and provide ATP that could be used during meiotic maturation, it was important to consider the possibility that increased metabolism of pyruvate, perhaps through the oxidative pathway under the conditions of high pyruvate concentration or ligand stimulation, plays an important role in the mechanisms regulating nuclear maturation of the oocyte. Therefore, in the present study, we have analysed the role of pyruvate in mouse oocyte maturation by: (I) examining the relationship between energy substrate utilization and oocyte maturation; and (2) measuring oxygen consumption under conditions of different substrate combinations and gonadotrophin stimulation.

\section{Materials and Methods}

\section{Oocyte isolation and culture conditions}

Experiments were carried out in Milwaukee, WI and York, UK, using two strains of mice: $\mathrm{C} 57 \mathrm{BL} / 6 \times \mathrm{SJL} / \mathrm{J} \mathrm{F}_{1}$ (B6SJL) in Milwaukee and $\mathrm{CBA} / \mathrm{Ca} \times \mathrm{C} 57 \mathrm{BL} / 6 \mathrm{~F}_{1}(\mathrm{CBAB} 6)$ in York. For each experiment, 20-23-day-old female mice were primed with 5 iu equine chorionic gonadotrophin (eCG) and killed $48 \mathrm{~h}$ later by cervical dislocation. The ovaries were removed, placed in medium, and antral follicles were punctured with sterile needles to release the oocyte-cumulus cell complexes. Complexes were washed and transferred to plastic culture tubes containing $1 \mathrm{mI}$ medium or to $7.5 \mu \mathrm{l}$ drops of medium under mineral oil in Falcon petri dishes.

The culture medium used was Eagle's minimum essential medium (MEM) containing Earle's salts, antibiotics, and $3 \mathrm{mg}$ lyophilized crystallized BSA ml ${ }^{-1}$ (ICN ImmunoBiologicals, from Lisle, IL or Than, UK). Concentrations of pyruvate and glucose varied depending on the particular experiment. For most of the experiments, medium was buffered by bicarbonate, and under these conditions was gassed with a humidified mixture of $5 \% \mathrm{CO}_{2}, 5 \% \mathrm{O}_{2}$ and $90 \% \mathrm{~N}_{2}$ to maintain $\mathrm{pH}$. For some experiments, including those involving measurement of oxygen consumption, bicarbonate was reduced to $0.5 \mathrm{~g} \mathrm{l}^{-1}$, Hepes was added at a concentration of $25 \mathrm{mmol} \mathrm{l}^{-1}$, and the $\mathrm{pH}$ was adjusted to 7.2 ; this medium was exposed to air.

\section{Energy substrate assays}

For the determination of energy substrate consumption or production, ten complexes were cultured in $7.5 \mu \mathrm{l}$ microdrops of medium under mineral oil for up to $18 \mathrm{~h}$. At the conclusion of culture, $6 \mu \mathrm{l}$ medium was removed and placed in a Cobas Mira assay tube. The oocytes were then scored for GVBD. Each medium sample was diluted with $124 \mu \mathrm{l}$ water and placed in a freezer at $-80^{\circ} \mathrm{C}$ until assayed. Medium samples were assayed for glucose, lactate and pyruvate concentrations using a Cobas Mira automated analyser (Roche Products Ltd, Welwyn Garden City), as described by Downs et al. (1996). The substrate measurements were performed at least three times with at least triplicate measurements per treatment group per experiment.

\section{Oxygen consumption}

Oxygen consumption by complexes was measured using a non-invasive method based on the fluorescent quaternary benzoid compound, pyrene, the fluorescence of which is quenched in the presence of oxygen (Houghton et al., 1996). Briefly, oxygen measurement was carried out in a $5 \mu \mathrm{l}$ PCR micropipette (Laser Laboratory Systems, Southampton) that contained $1 \mu \mathrm{l}$ pyrene at a concentration of $1 \mathrm{mmol} \mathrm{l}^{-1}$ (Sigma Chemical Co., Poole) dissolved in paraffin oil (BDH, Poole) and $2 \mu \mathrm{l}$ medium containing two-three oocyte-cumulus cell complexes (see details in Houghton et al., 1996). Oxygen consumption was measured by following the increase in pyrene fluorescence at $340 \mathrm{~nm}$ over a $3-5 \mathrm{~h}$ period on a Fluovert fluorescence microscope (Leica UK Ltd, Milton Keynes) with photomultiplier and photometer attachments. For each experiment, two controls were used: (1) a $0 \%$ oxygen control containing $1 \mu \mathrm{l}$ pyrene and $2 \mu \mathrm{l}$ of yeast $\left(1 \mathrm{mg} \mathrm{ml}^{-1}\right)$ in glucose $\left(60 \mathrm{mmol} \mathrm{l}^{-1}\right)$ pre-equilibrated overnight; and (2) a $20 \%$ oxygen control containing $1 \mu \mathrm{l}$ pyrene and $2 \mu \mathrm{l}$ Hepesbuffered MEM-BSA saturated with oxygen but in the absence of complexes.

\section{Chemicals}

All media components, hypoxanthine, dichloroacetate (DCA) and Hepes were obtained from Sigma Chemical Co. (St Louis, MO or Poole). FSH was a gift from the National Hormone and Pituitary Program of the NIDDK (ovine FSH-20, concentration in medium of $0.01 \mu \mathrm{g} \mathrm{ml}^{-1}$; Bethesda, MD) or was purchased from ImmunoChemical Products (Auckland,; Ovagen, concentration in medium of $0.5 \mu \mathrm{g} \mathrm{ml}^{-1}$ ). eCG was purchased from Diosynth Inc. (Chicago, IL) or from Intervet (Cambridge, UK, as Folligon).

\section{Statistical analysis}

Each oocyte maturation experiment was carried out a minimum of three times, with at least 40 oocytes per treatment group per experiment. Results are presented as the mean percentage frequency of GVBD occurring in oocytes plus or minus the standard error of the mean, and were subjected to arcsine transformation before statistical analysis. Transformed 
oocyte maturation data and non-transformed oxygen consumption and energy substrate consumption or production data were analysed by ANOVA followed by Duncan's multiple range test. Paired comparisons were made by Student's $t$ test. A probability of $P<0.05$ was considered significant.

\section{Results}

Effects of culture conditions on oocyte maturation and energy substrate dynamics

An initial experiment was carried out in which cumulus cell-enclosed oocytes were cultured for 9 or $18 \mathrm{~h}$ in $7.5 \mu \mathrm{l}$ drops of medium supplemented with $4 \mathrm{mmol}$ hypoxanthine $1^{-1}$ containing $0.23 \mathrm{mmol}$ pyruvate $\mathrm{l}^{-1}$ or $1 \mathrm{mmol}$ pyruvate $\mathrm{l}^{-1}$ alone, or $1 \mathrm{mmol}$ pyruvate $\mathrm{l}^{-1}$ plus $5.5 \mathrm{mmol}$ glucose $\mathrm{l}^{-1}$. At the end of culture, medium was assayed for pyruvate, while the meiotic status of the oocytes was assessed. No differences in maturation frequency were observed at $9 \mathrm{~h}$, with GVBD occurring in 16-24\% of the oocytes (Fig. Ia). However, by $18 \mathrm{~h}, 82 \%$ of oocytes in the group cultured in $1 \mathrm{mmol}$ pyruvate $1^{-1}$ had undergone maturation, compared with only $37 \%$ in the group cultured in $0.23 \mathrm{mmol}$ pyruvate $\mathrm{I}^{-1}$. The inclusion of $5.5 \mathrm{mmol}$ glucose $1^{-1}$ prevented the increase in maturation brought about by 1 mmol pyruvate $\mathrm{l}^{-1}$ (44\% GVBD).

Pyruvate assays showed that by $9 \mathrm{~h}$ of culture pyruvate consumption by complexes was more than doubled in the cultures with a higher pyruvate concentration $10.203 \mathrm{nmol}$ per complex in $1 \mathrm{mmol}$ pyruvate $1^{-1}$ compared with $0.094 \mathrm{nmol}$ per complex in $0.23 \mathrm{mmol}$ pyruvate $\mathrm{I}^{-1}$; Fig. 1b). This relationship continued throughout the following $9 \mathrm{~h}$ (by $18 \mathrm{~h}$ of culture, pyruvate consumption was $0.37 \mathrm{nmol}$ per complex in I nmol pyruvate $1^{-1}$ compared with $0.19 \mathrm{nmol}$ per complex in $0.23 \mathrm{mmol}$ pyruvate $1^{-1}$ ). The addition of glucose to medium containing $1 \mathrm{mmol}$ pyruvate $1^{-1}$ prevented the increase in pyruvate consumption brought about by the higher concentration of pyruvate (Fig. Ib).

The decrease in pyruvate consumption upon addition of glucose to culture medium reveals the ability of one energy substrate to modify the consumption of another. This relationship was examined in greater detail by culturing cumulus cell-enclosed oocytes for $18 \mathrm{~h}$ in microdrops in medium containing $4 \mathrm{mmol}$ hypoxanthine $\mathrm{I}^{-1}$ plus one of five energy substrate supplementations: (1) $0.23 \mathrm{mmol}$ pyruvate $\mathrm{l}^{-1}$; (2) I mmol pyruvate $\mathrm{l}^{-1}$; (3) $5.5 \mathrm{mmol}$ glucose $1^{-1}$; (4) $0.23 \mathrm{mmol}$ pyruvate $\mathrm{I}^{-1}$ plus $5.5 \mathrm{mmol}$ glucose $\mathrm{l}^{-1}$; or (5) $1 \mathrm{mmol}$ pyruvate $\mathrm{I}^{-1}$ plus $5.5 \mathrm{mmol}$ glucose $\mathrm{I}^{-1}$. Oocytes were then scored for GVBD and the medium was analysed for substrate composition. In the absence of glucose, $24 \%$ and $67 \%$ of oocytes underwent GVBD in 0.23 and 1 mmol pyruvate $1^{-1}$, respectively (Fig. 2a). The addition of glucose to medium containing $0.23 \mathrm{mmol}$ pyruvate $\mathbb{1}^{-1}$ had no effect on maturation but in medium containing the higher pyruvate concentration, glucose reduced the frequency of maturation by half (to $32 \%$ GVBD). The highest percentage of maturation was observed in the group cultured in glucose alone $(87 \%$ GVBD).

Consistent with the results shown in Fig. 1, complexes cultured in pyruvate alone consumed this substrate $(0.136$ and $0.383 \mathrm{nmol}$ per complex in 0.23 and $1 \mathrm{mmol}$ pyruvate $\mathrm{l}^{-1}$.
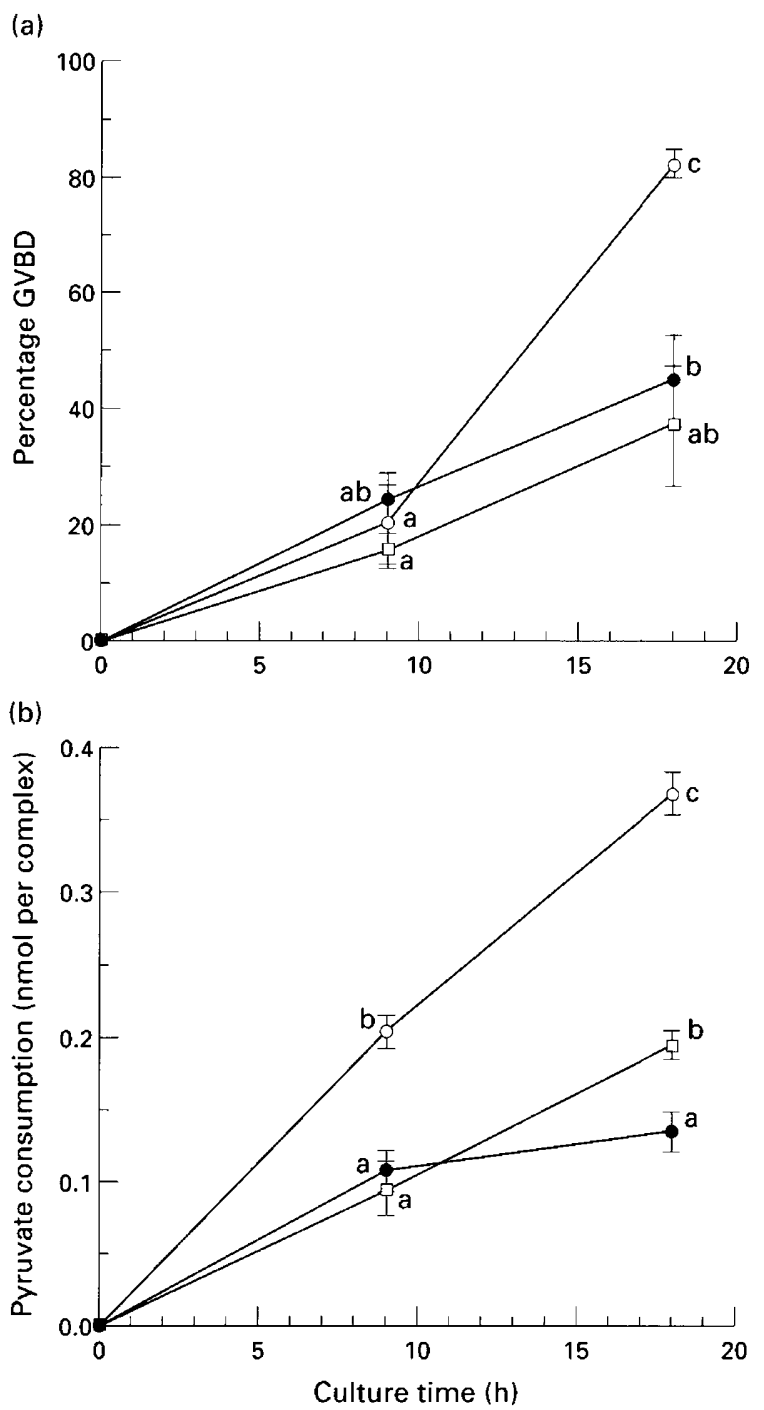

Fig. 1. The effects of pyruvate and glucose on the maturation of mouse oocytes and pyruvate consumption in the culture medium. Cumulus cell-enclosed oocytes were cultured in microdrops for 9 or $18 \mathrm{~h}$ in medium containing $4 \mathrm{mmol}$ hypoxanthine $\mathrm{l}^{-1}$ plus $0.23 \mathrm{mmol}$ pyruvate $\mathrm{I}^{-1}(\square), 1 \mathrm{mmol}$ pyruvate $\mathrm{l}^{-1}(O)$ or $1 \mathrm{mmol}$ pyruvate $\mathrm{l}^{-1}$ plus $5.5 \mathrm{mmol}$ glucose $\mathrm{I}^{-1}$ ( ). At the end of the culture period (a) oocytes were assessed for germinal vesicle breakdown (GVBD) and (b) medium was analysed for pyruvate consumption. In (a) and (b), groups with at least one identical letter are not signficantly different.

respectively; Fig. 2b). Addition of glucose to medium containing $1 \mathrm{mmol}$ pyruvate $1^{-1}$ reduced consumption of pyruvate by $52 \%$ (to $0.182 \mathrm{nmol}$ per complex), while addition of glucose to medium containing $0.23 \mathrm{mmol}$ pyruvate $1^{-1}$ led to a net appearance of pyruvate $(0.211 \mathrm{nmol}$ per complex). The highest glucose consumption occurred in the group cultured in glucose alone $(2.058 \mathrm{nmol}$ per complex; Fig. $2 \mathrm{~b})$, and the addition of pyruvate to glucose-containing medium resulted in a reduction in glucose consumption $(0.482 \mathrm{nmol}$ per complex consumed in the presence of 1 nmol pyruvate $1^{-1}$; a decrease of $77 \%$ ). Lactate accumulated in the three glucose-containing groups, and the amount generated was related to the amount of glucose consumed (Fig. 2b). Lactate also appeared in the group 


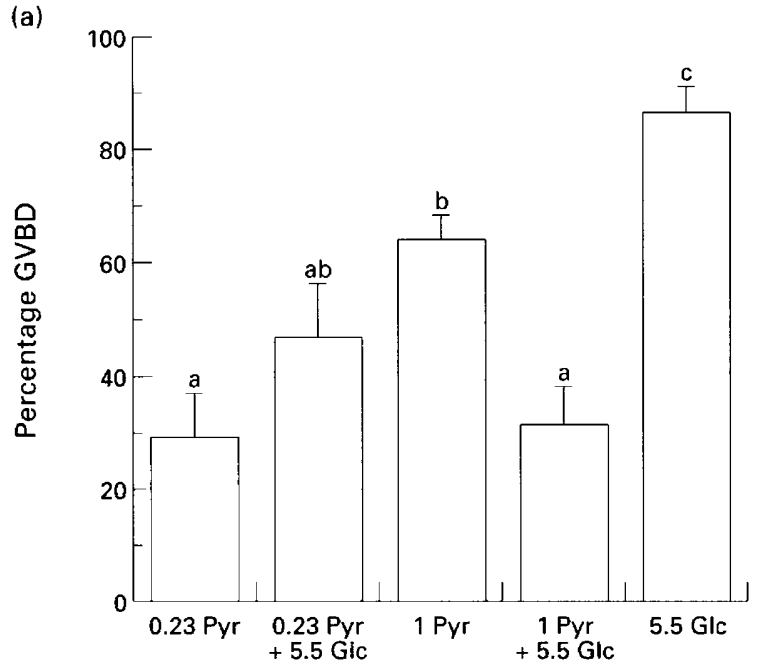

(b)

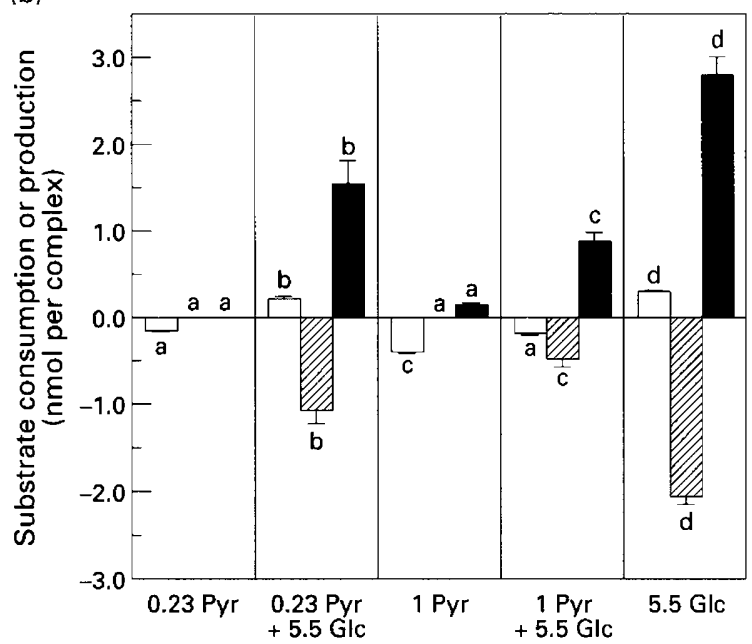

Fig. 2. The effects of different combinations of pyruvate (Pyr) and glucose (Glc) on mouse oocyte maturation and substrate concentrations in the culture medium. Cumulus cell-enclosed oocytes were cultured in microdrops for $18 \mathrm{~h}$ in medium containing $4 \mathrm{mmol}$ hypoxanthine $\mathrm{I}^{-1}$ plus different millimolar concentrations of pyruvate or glucose. Oocytes were then assessed for germinal vesicle breakdown (GVBD; a) and medium was analysed for pyruvate ( $\square$ ), glucose (E) and lactate ( $\square$ ) concentrations (b). In (b), a negative value denotes consumption, while a positive value represents net accumulation of substrate. Statistical analyses were carried out separately for each energy substrate. In (a) and (b), groups with at least one identical letter are not significantly different.

cultured in $1 \mathrm{mmol}$ pyruvate $\mathrm{I}^{-1}$ and could account for $42 \%$ of the pyruvate consumed.

The high frequency of maturation in medium containing only $5.5 \mathrm{mmol}$ glucose $\mathrm{l}^{-1}$ was inconsistent with our previous finding (Downs and Mastropolo, 1994) that increasing concentrations of glucose up to $2.3 \mathrm{mmol}^{-1}$ had no effect on hypoxanthine-maintained meiotic arrest in the absence of pyruvate. However, since that study was carried out with oocytes cultured in I $\mathrm{ml}$ of medium in tubes instead of microdrops, we performed an experiment that compared the maturation of cumulus cell-enclosed oocytes in tubes and microdrops when treated with hypoxanthine under various (a)

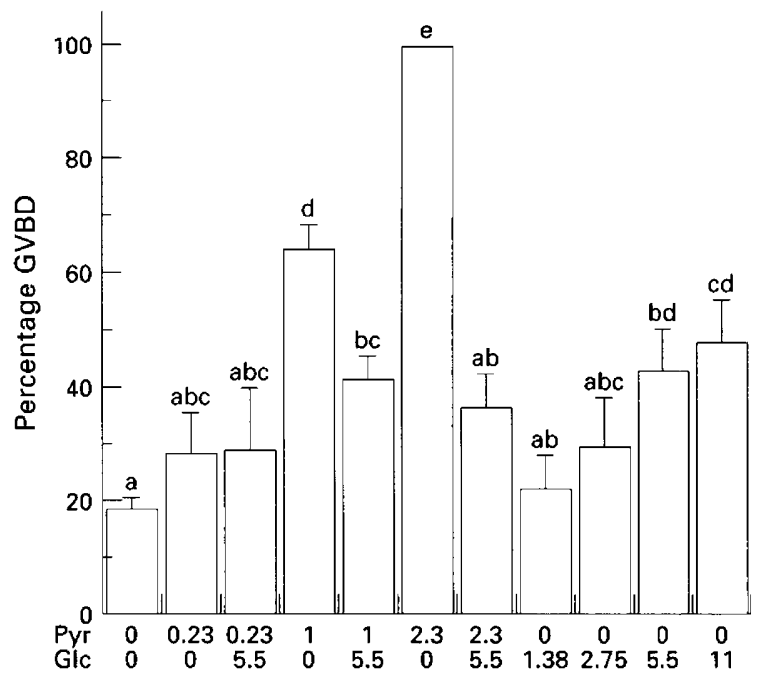

(b)

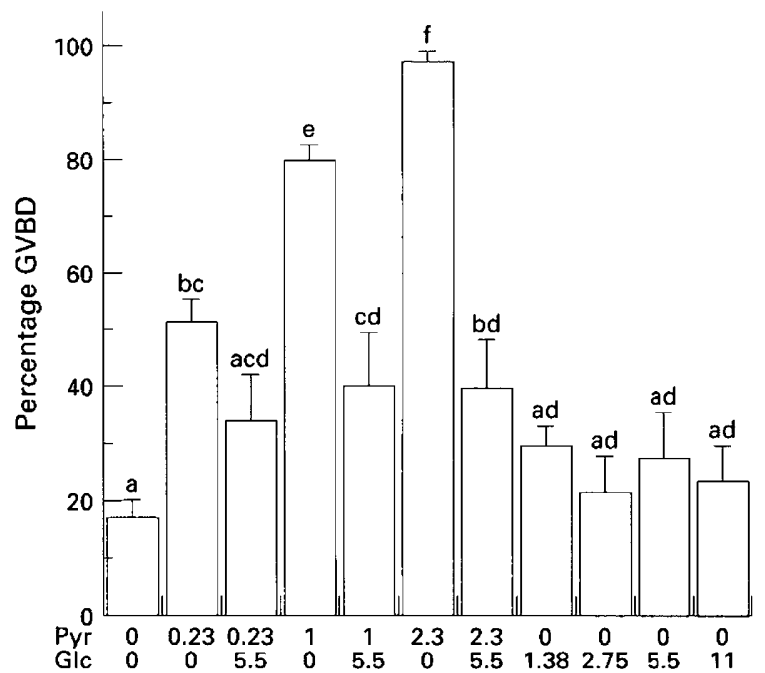

Fig. 3. Comparison of substrate effects on mouse oocyte maturation. Cumulus cell-enclosed oocytes were cultured for $17-18 \mathrm{~h}$ in medium containing $4 \mathrm{mmol}$ hypoxanthine $1^{-1}$ plus different millimolar concentrations of pyruvate (Pyr) and glucose (Glc) in (a) $7.5 \mu \mathrm{l}$ microdrops under oil or (b) in tubes containing $1 \mathrm{ml}$ of medium. Groups with at least one identical letter are not significantly different.

energy substrate combinations. When neither pyruvate nor glucose was included in the culture medium, maturation frequencies were equivalent in the two groups (17-18\%; Fig. 3). Under both types of culture condition, a dose-dependent increase in GVBD was observed when pyruvate alone was added to the medium. The frequency of maturation was higher in oocytes cultured in tubes in 0.23 and $1 \mathrm{mmol}$ pyruvate $1^{-1}$, but $\geq 97 \%$ GVBD was observed in both groups cultured in $2.3 \mathrm{mmol}$ pyruvate $1^{-1}$. Increasing concentrations of glucose alone had no effect on the maturation in oocytes cultured in tubes, but a dose-dependent increase was observed in microdrop cultures, with maturation in $11 \mathrm{mmol}$ glucose $\mathrm{I}^{-1}$ occurring at a frequency of $48 \%$ (compared with $18 \%$ in the absence of glucose and pyruvate). Consistent with the previous experiment, the addition of $5.5 \mathrm{mmol}$ glucose $\mathrm{I}^{-1}$ to pyruvatecontaining medium suppressed the maturation induced by the 
higher concentrations of pyruvate; maturation frequencies were similar regardless of the pyruvate concentration or whether oocytes were cultured in tubes or microdrops.

Increased pyruvate utilization is not required for resumption of meiotic maturation

Experiments were next carried out to test whether an increase in pyruvate consumption regulates resumption of meiotic maturation. Although the above data indicate that increased pyruvate utilization may be associated with increased oocyte maturation, an earlier study (Downs et al., 1996) also showed that pyruvate production by hypoxanthine-arrested oocytes treated with FSH reached a plateau at the time hormone-induced maturation was being initiated. This result from the earlier study suggested that a switch from pyruvate production to consumption might be occurring after reinitiation of meiotic maturation. It was therefore conceivable that an increase in pyruvate consumption is not the cause of, but rather an indicator of, GVBD.

This idea was tested by culturing oocyte-cumulus cell complexes for $3 \mathrm{~h}$ in microdrops containing MEM supplemented with $5.5 \mathrm{mmol}$ glucose $1^{-1}$ and $0.23 \mathrm{mmol}$ pyruvate $\mathrm{l}^{-1}(0-3 \mathrm{~h}$ culture). A second group of complexes was first cultured for $3 \mathrm{~h}$ in tubes containing $1 \mathrm{ml}$ of the same medium to permit GVBD and then transferred to microdrops for $3 \mathrm{~h}$ (4-6 h culture). Preliminary experiments established that after $3 \mathrm{~h}$ of culture $>93 \%$ of the oocytes had undergone GVBD. After culture in microdrops for $3 \mathrm{~h}, 6 \mu \mathrm{l}$ medium was collected from each microdrop and analysed for glucose, lactate and pyruvate concentrations. No net change in the concentration of pyruvate was evident in the group cultured for up to $3 \mathrm{~h}$, whereas each complex consumed $0.166 \mathrm{nmol}$ glucose and produced $0.173 \mathrm{nmol}$ lactate (Fig. 4). The substrate profile was significantly altered in the group cultured for 4-6 h. Glucose consumption per complex was reduced $83 \%$ to $0.029 \mathrm{nmol}$, with a corresponding $88 \%$ reduction in lactate production (to $0.020 \mathrm{nmol})$. However, pyruvate was consumed during this period, at a rate of $0.022 \mathrm{nmol}$ per complex (Fig. 4).

Microdrop cultures were maintained for $18 \mathrm{~h}$ in medium containing $0.46 \mathrm{mmol}$ pyruvate $1^{-1}, 5.5 \mathrm{mmol}$ glucose $1^{-1}$, and $4 \mathrm{mmol}$ hypoxanthine $\mathrm{l}^{-1}$ and were treated with increasing concentrations of dichloroacetate (DCA), a compound known to stimulate the activity of pyruvate dehydrogenase and, thus, increase pyruvate consumption (Whitehouse et al., 1974), to determine whether stimulation of pyruvate consumption would increase GVBD in hypoxanthine-arrested cumulus cell-enclosed oocytes. The medium was assayed for pyruvate, glucose and lactate, and oocytes were scored for GVBD. In the absence of DCA, each complex consumed $0.014 \mathrm{nmol}$ pyruvate but this value increased ninefold with the addition of DCA, so that at a concentration of $1 \mathrm{mmol}$ pyruvate $\mathrm{I}^{-1}$, consumption had reached $0.129 \mathrm{nmol}$ per complex (Fig. 5a). In contrast, glucose consumption and lactate production tended to decrease in response to DCA treatment (Fig. 5a). Despite the increase in pyruvate utilization, DCA had no discernible effect on oocyte maturation, the frequency of GVBD ranging from 14 to $23 \%$ (Fig. 5b). These data indicate that stimulating the consumption of pyruvate does not necessarily stimulate oocyte maturation.

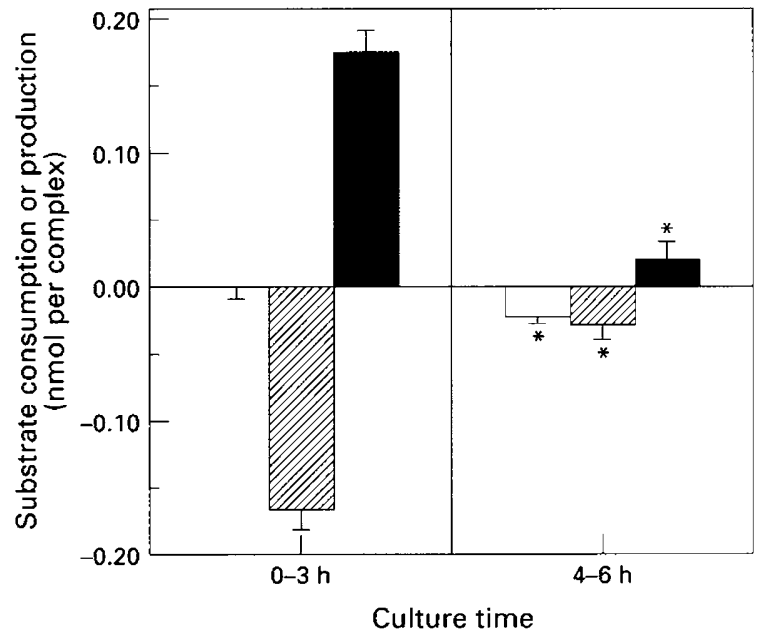

Fig. 4. Substrate concentrations during spontaneous mouse oocyte maturation. Cumulus cell-enclosed oocytes were cultured in microdrops for $3 \mathrm{~h}$ in inhibitor-free medium either immediately after isolation or after an initial $3 \mathrm{~h}$ culture in tubes to permit germinal vesicle breakdown (GVBD). Medium was then assayed for pyruvate $(\square)$, glucose $(\square)$ and lactate $(\square)$ concentrations. A negative value denotes consumption, while a positive value represents net accumulation. An asterisk denotes a significant difference from the corresponding group cultured for $0-3 \mathrm{~h}(P<0.05)$.

In a previous study (Downs and Mastropolo, 1994), it was shown that FSH had only a nominal stimulatory effect on the maturation of hypoxanthine-arrested oocytes in glucose-free medium. In the present study, we performed a kinetic analysis of pyruvate consumption by hypoxanthine-arrested, cumulus cell-enclosed oocytes cultured in glucose-free microdrops of medium for 6,9 and $18 \mathrm{~h}$ in the presence or absence of FSH. At none of the times analysed was there a difference in pyruvate utilization between the groups cultured in the presence or absence of FSH (Fig. 6).

An additional experiment tested the effects of two different concentrations of pyruvate on FSH-induced maturation and pyruvate consumption in microdrop cultures. Cumulus cellenclosed oocytes were cultured for $18 \mathrm{~h}$ in hypoxanthinesupplemented, glucose-free medium containing either 0.23 or $0.46 \mathrm{mmol}$ pyruvate $\mathrm{I}^{-1}$ in the presence or absence of FSH. In each of these conditions, comparable numbers of oocytes underwent GVBD (Fig. 7a). Pyruvate consumption by complexes was doubled in the groups cultured in the higher concentration of pyruvate, regardless of the presence or absence of FSH (Fig. 7b). Therefore, these data fail to support the contention that FSH induction of maturation is brought about by increased pyruvate consumption.

\section{Relationship between oxygen consumption and oocyte maturation}

The possible participation of TCA cycle activity in the regulation of meiotic maturation was evaluated by measuring oxygen consumption by complexes under different culture conditions. To carry out these experiments, we first needed a culture system that did not rely on a $\mathrm{CO}_{2}$ gas phase to maintain $\mathrm{pH}$. In a recent study, we showed that when the bicarbonate in MEM is reduced from $26 \mathrm{mmol} \mathrm{I}^{-1}$ to $6 \mathrm{mmol}$ 
(a)

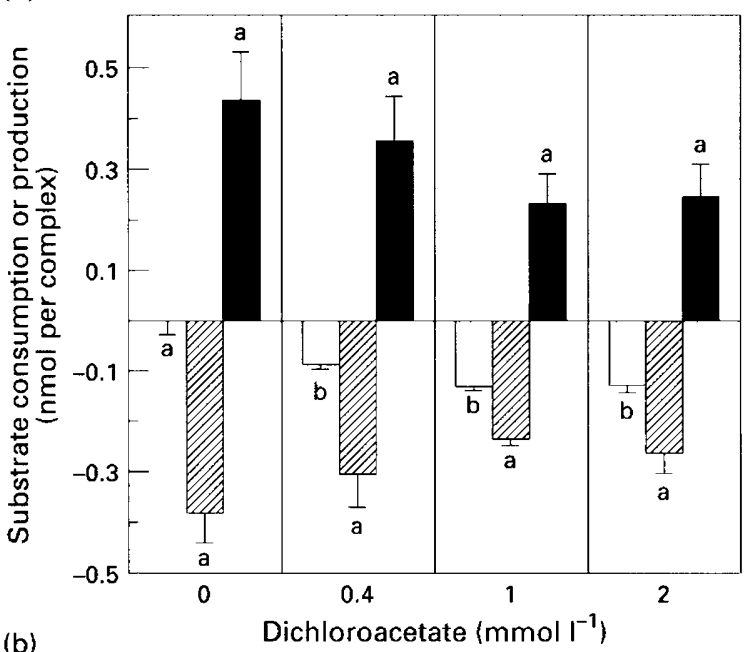

(b)

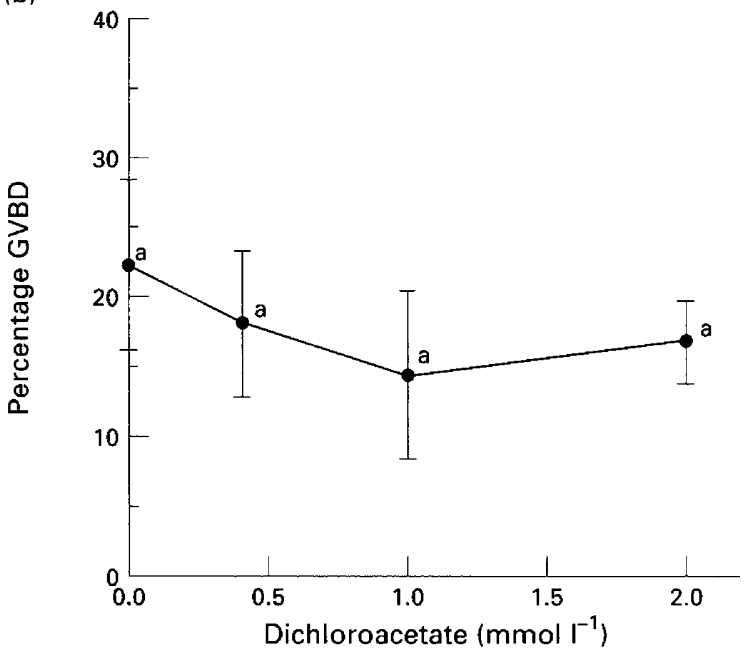

Fig. 5. The effects of dichloroacetate on hypoxanthine-maintained meiotic arrest in mouse oocytes and substrate concentrations in the culture medium. Cumulus cell-enclosed oocytes were cultured in microdrops for $18 \mathrm{~h}$ in medium containing $4 \mathrm{mmol}$ hypoxanthine $\mathrm{l}^{-\mathrm{I}}$ plus $0.46 \mathrm{mmol}$ pyruvate $\mathrm{I}^{-1}$ and $5.5 \mathrm{mmol}$ glucose $\mathrm{l}^{-1}$. At the end of the culture period, (a) medium was assayed for pyruvate ( $\square$ ), glucose ( $\square$ ) and lactate ( $\boldsymbol{\square}$ ) concentrations and (b) oocytes were assessed for germinal vesicle breakdown (GVBD). In (a), a negative value denotes consumption, while a positive value represents net accumulation. Statistical analyses were carried out separately for each energy substrate. In (a) and (b), groups with the same letter are not significantly different.

$1^{-1}$ and $25 \mathrm{mmol}$ Hepes $\mathrm{1}^{-1}$ is added, the $\mathrm{pH}$ can be maintained at 7.2 during overnight culture in the absence of a $\mathrm{CO}_{2}$ gas phase and the system supports FSH-induced maturation in hypoxanthine-arrested oocytes (Downs and Mastropolo, in press). Since these previous experiments were performed with a large volume of medium $(1 \mathrm{ml})$ in culture tubes, and since data presented here show that the volume of medium can have a significant impact on meiotic behaviour (see Fig. 3), it was important to establish that a similar meiotic response would be manifested in microlitre volumes and to compare the energy substrate dynamics under conditions of Hepes buffering with those in the bicarbonate-buffered system.

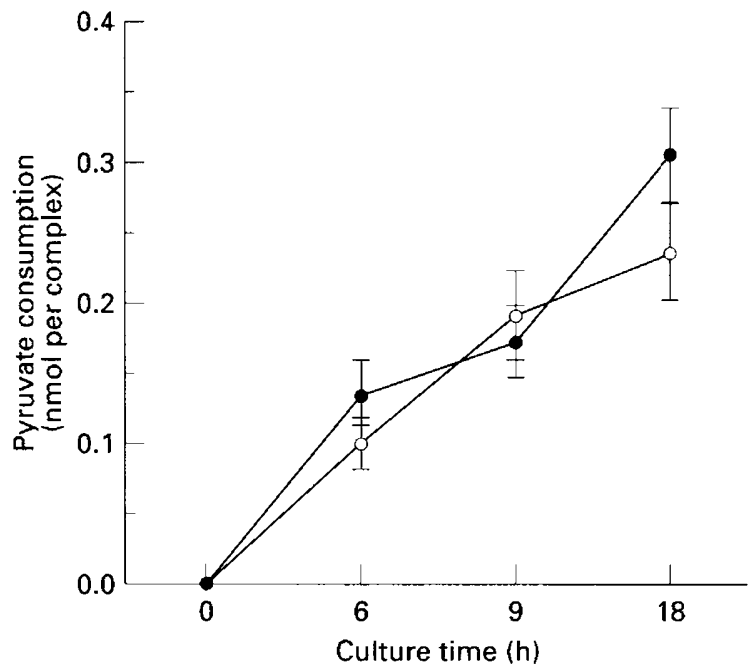

Fig. 6. The effects of FSH on pyruvate consumption in glucose-free medium. Cumulus cell-enclosed mouse oocytes were cultured in microdrops for 6,9 or $18 \mathrm{~h}$ in medium containing $4 \mathrm{mmol}$ hypoxanthine $1^{-1}$ plus $0.23 \mathrm{mmol}$ pyruvate $\mathrm{l}^{-1}$ and $5.5 \mathrm{mmol}$ glucose $\mathrm{I}^{-1}$, in the presence ( $)$ or absence $(O)$ of FSH. Data represent the amount of pyruvate consumed per oocyte-cumulus cell complex after the appropriate culture period.

In the absence of Hepes (bicarbonate-buffered medium), $40 \%$ of cumulus cell-enclosed oocytes cultured in microdrops of medium containing $4 \mathrm{mmol}$ hypoxanthine $\mathrm{I}^{-1}$ underwent GVBD in the absence of FSH; treatment with FSH increased this number to $99 \%$ (Fig. 8). When medium was buffered with Hepes, only $5 \%$ of the oocytes resumed maturation, but FSH stimulation induced nearly all of the oocytes to undergo maturation (97\%).

In bicarbonate-buffered medium, complexes consumed glucose at a rate of $0.87 \mathrm{nmol}$ per complex in the absence of FSH and $2.79 \mathrm{nmol}$ per complex in its presence. In Hepes-buffered medium, less glucose was used, with $0.57 \mathrm{nmol}$ consumed per complex in the absence of FSH and 1.58 nmol per complex in its presence. Pyruvate and lactate production were also reduced in Hepes-buffered cultures, in parallel with the change in glucose consumption. These results demonstrate that the Hepes-buffered system is an excellent way of both maintaining meiotic arrest with hypoxanthine and supporting FSHinduced meiotic maturation, although glucose metabolism is attenuated.

Two types of experiments were carried out to examine oxygen consumption of oocyte-cumulus cell complexes under conditions that stimulate meiotic maturation. First, the effects of FSH were tested at various intervals after the initiation of culture. Cumulus cell-enclosed oocytes were cultured for 0,4 or $8 \mathrm{~h}$ in tubes in $1 \mathrm{ml}$ of Hepes-buffered medium containing $4 \mathrm{mmol}$ hypoxanthine $\mathrm{l}^{-1}, 5.5 \mathrm{mmol}$ glucose $\mathrm{l}^{-1}$ and 0.23 mmol pyruvate $\mathrm{l}^{-1}$, plus or minus $\mathrm{FSH}$, and then were transferred in $2 \mu \mathrm{l}$ of the same medium to a capillary pipette for measurement of oxygen consumption. Oxygen consumption by complexes was significantly less in groups exposed to FSH, regardless of the preincubation period in tubes, and was still suppressed at the time that induction of GVBD was initiated (Table 1). 
(a)

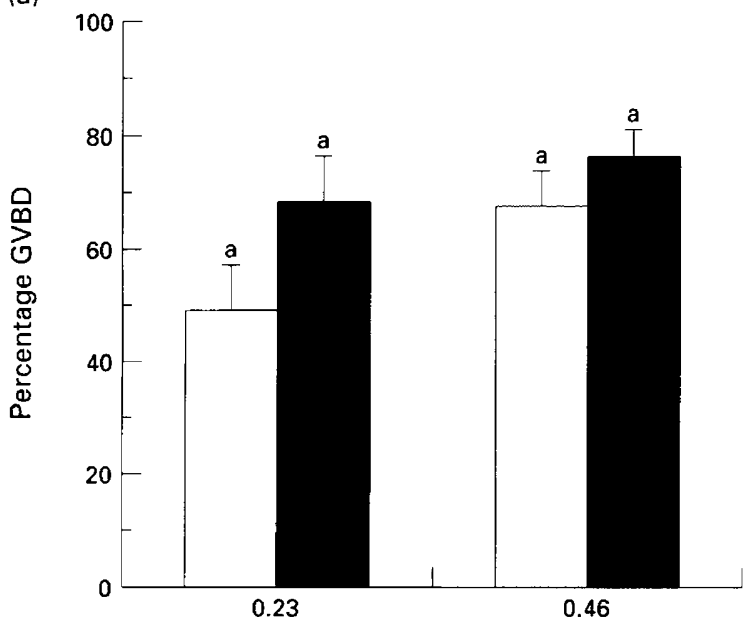

(b)

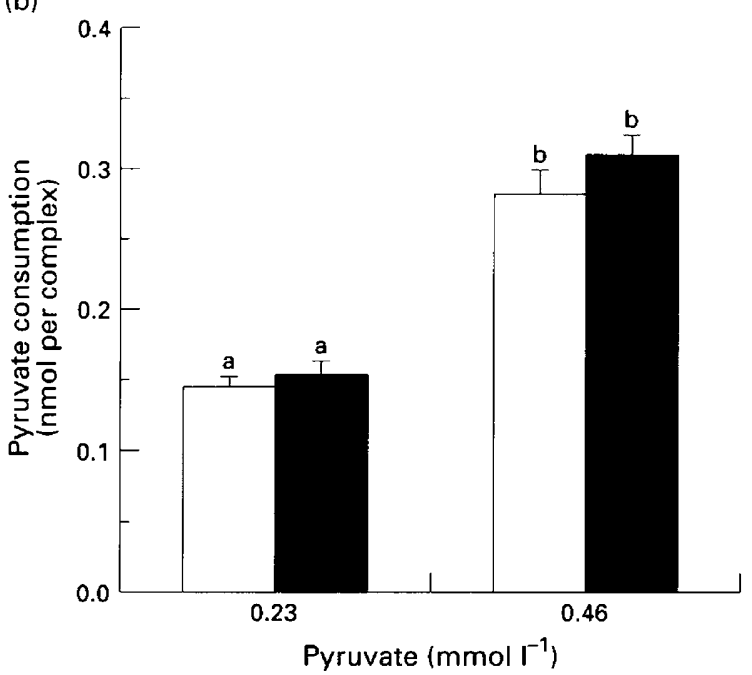

Fig. 7. The effects of different pyruvate concentrations and FSH on mouse oocyte maturation and pyruvate consumption in the culture medium. Cumulus cell-enclosed oocytes were cultured in microdrops for $18 \mathrm{~h}$ in glucose-free medium containing $4 \mathrm{mmol}$ hypoxanthine $\mathrm{I}^{-1}$ plus 0.23 or $0.46 \mathrm{mmol}$ pyruvate $\mathrm{I}^{-1}$, in the presence $(\boldsymbol{D})$ or absence ( $\square$ ) of FSH. (a) Oocytes were then assessed for germinal vesicle breakdown (GVBD) and (b) medium was analysed for pyruvate consumption. In (a) and (b), groups with the same letter are not significantly different.

The second experiment involved culturing complexes for $4 \mathrm{~h}$ in medium containing hypoxanthine plus one of three energy substrate conditions: (1) $0.23 \mathrm{mmol}$ pyruvate $\mathrm{l}^{-1}$; (2) $1 \mathrm{mmol}$ pyruvate $\mathrm{1}^{-1}$; or (3) $1 \mathrm{mmol}$ pyruvate $\mathrm{1}^{-1}$ plus $5.5 \mathrm{mmol}$ glucose $\mathrm{I}^{-1}$. At the end of the preincubation period, complexes were then placed in $2 \mu \mathrm{l}$ of the same medium in capillary pipettes for the measurement of oxygen consumption. The highest oxygen consumption occurred in medium supplemented with $0.23 \mathrm{mmol}$ pyruvate $\mathrm{l}^{-1}(5.96 \mathrm{nl}$ per complex $\mathrm{h}^{-1}$; Table 2), less oxygen being consumed at the higher pyruvate concentration $\left(4.69 \mathrm{nl}\right.$ per complex $\mathrm{h}^{-1}$ ). The addition of glucose produced the lowest oxygen consumption $\left(3.78 \mathrm{nl}\right.$ per complex $\left.\mathrm{h}^{-1}\right)$. These data indicate that glucose can lower the rate of oxygen consumption but fail to support the contention that the increased maturation frequency in response (a)

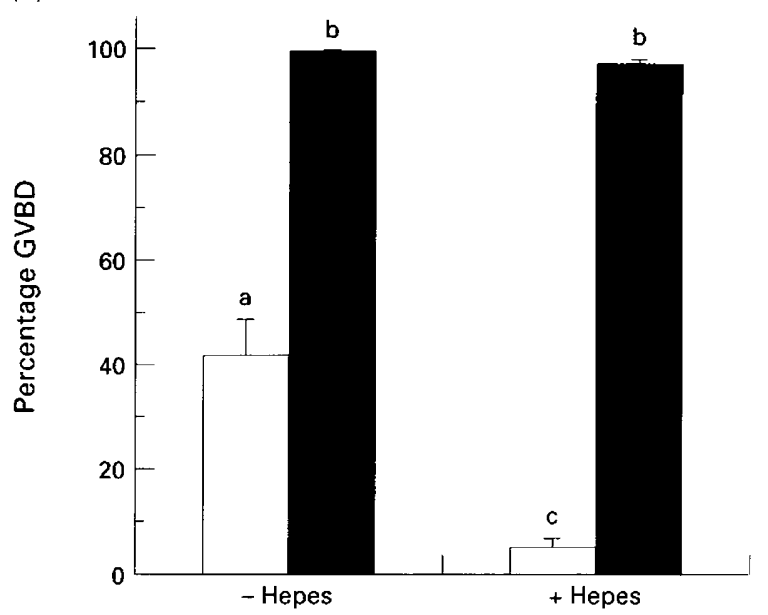

(b)

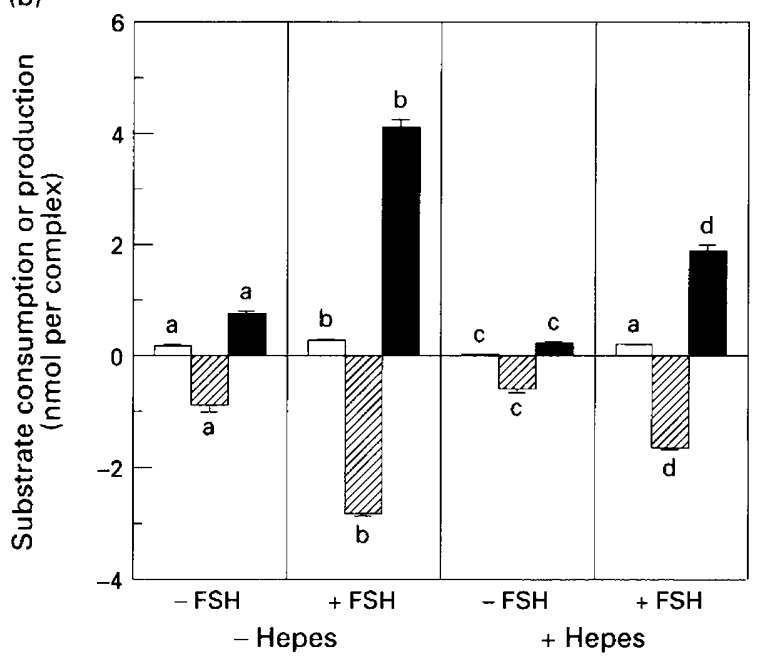

Fig. 8. Effects of Hepes buffer on mouse oocyte maturation and substrate concentrations in the culture medium. Cumulus cell-enclosed oocytes were cultured in microdrops for $18 \mathrm{~h}$ in bicarbonate-buffered ( - Hepes) or Hepes-buffered ( + Hepes) medium containing $4 \mathrm{mmol}$ hypoxanthine $1^{-1}$, in the presence or absence of FSH. (a) Oocytes were then assessed for germinal vesicle breakdown (GVBD) $(\square,-$ FSH; $\square,+$ FSH) and (b) medium was analysed for pyruvate ( $\square$ ), glucose $(\mathbb{Z})$ and lactate $(\boldsymbol{\square})$ concentrations. In (b), a negative value denotes consumption, while a positive value represents net accumulation. Statistical analyses were carried out separately for each energy substrate. In (a) and (b), groups with the same letter are not significantly different.

to high concentrations of pyruvate or $\mathrm{FSH}$ is due to oxidation of pyruvate through the TCA cycle.

\section{Discussion}

This study reports two principal findings regarding energy substrate dynamics and the control of oocyte maturation. First, pyruvate and glucose metabolism by the oocyte-cumulus cell complex is under at least partial control by the complementary substrate, and this can have a marked impact on oocyte maturation. High concentrations of either pyruvate or glucose alone may exhibit a meiosis-inducing effect on inhibitor-treated 
Table 1. Effect of FSH on germinal vesicle breakdown (GVBD) and oxygen consumption in mouse oocyte-cumulus cell complexes

\begin{tabular}{|c|c|c|c|c|}
\hline \multirow[b]{2}{*}{ Treatment } & \multicolumn{2}{|c|}{$-\mathrm{FSH}$} & \multicolumn{2}{|c|}{$+\mathrm{FSH}$} \\
\hline & GVBD & $\begin{array}{l}\text { Oxygen } \\
\text { consumed }\end{array}$ & GVBD & $\begin{array}{l}\text { Oxygen } \\
\text { consumed }\end{array}$ \\
\hline $\mathrm{Oh}$ & 0 & $\begin{array}{c}3.46 \pm 0.28 \\
\text { (14) }\end{array}$ & 0 & $\begin{array}{c}2.75 \pm 0.17^{*} \\
(16)\end{array}$ \\
\hline $4 \mathrm{~h}$ & 3.9 & $\begin{array}{c}4.31 \pm 0.28 \\
\text { (15) }\end{array}$ & 0 & $\begin{array}{c}3.72 \pm 0.22^{*} \\
(16)\end{array}$ \\
\hline $8 \mathrm{~h}$ & 2.8 & $\begin{array}{c}4.76 \pm 0.22 \\
(16)\end{array}$ & 10.7 & $\begin{array}{c}4.11 \pm 0.31 * \\
(14)\end{array}$ \\
\hline $18 \mathrm{~h}$ & 14.2 & ND & 87.9 & ND \\
\hline
\end{tabular}

Complexes were cultured for 0,4 , or $8 \mathrm{~h}$ in medium containing $4 \mathrm{mmol}$ hypoxanthine $l^{-1} \pm \mathrm{FSH}$ and then assayed in the same medium for oxygen consumption (nl per complex $\mathrm{h}^{-1}$ ). The numbers in parentheses represent the number of assays for each group. An asterisk denotes a significant difference from the corresponding - FSH group. GVBD was assessed after $0,4,8$ or $18 \mathrm{~h}$ of culture, and data are presented as the mean percentage of four determinations.

ND, not determined.

Table 2. Effect of energy substrates on oxygen consumption in mouse oocyte-cumulus cell complexes

Oxygen consumed

Treatment

(nl per complex $\mathrm{h}^{-1}$ )

$0.23 \mathrm{mmol}$ pyruvate $\mathrm{I}^{-1}$
$1 \mathrm{mmol}$ pyruvate $\mathrm{l}^{-1}$
$1 \mathrm{mmol}$ pyruvate $\mathrm{l}^{-1}$
$+5.5 \mathrm{mmol}_{\text {glucose }} \mathrm{l}^{-1}$

$5.96 \pm 0.21^{\mathrm{a}}$

(6)

$4.69 \pm 0.64^{\mathrm{ab}}$

(6)

$3.78 \pm 0.54^{b}$

(6)

Complexes were cultured for $4 \mathrm{~h}$ in medium containing $4 \mathrm{mmol}$ hypoxanthine $\mathrm{1}^{-1}$ in one of three energy substrate supplementation groups: $0.23 \mathrm{mmol}$ pyruvate $\mathrm{I}^{-1}$; $1 \mathrm{mmol}$ pyruvate $\mathrm{I}^{-1}$; or $1 \mathrm{mmol}$ pyruvate $\mathrm{I}^{-1}$ plus $5.5 \mathrm{mmol}$ glucose $1^{-1}$. They were then assayed in the same medium for oxygen consumption. The numbers in parentheses represent the number of assays for each group. Groups with at least one identical letter are not significantly different.

oocytes, and this is accompanied by high rates of substrate utilization, but addition of the other substrate can significantly negate both the meiotic induction and high rate of substrate consumption. Second, pyruvate oxidation is apparently not an important component of the meiotic induction process that occurs by either energy substrate manipulation or by hormone stimulation. Although under certain culture conditions increased consumption of pyruvate preceded an increase in GVBD, subsequent experimentation failed to confirm a consistent causal relationship between increased pyruvate or oxygen consumption and meiotic resumption.

The initial kinetic experiment with hypoxanthine-treated, cumulus cell-enclosed oocytes demonstrated that twice as much pyruvate was consumed in medium containing $1 \mathrm{mmol}$ pyruvate $l^{-1}$ as in that containing $0.23 \mathrm{mmol}$ pyruvate $I^{-1}$.
Moreover, this increased consumption preceded a stimulatory effect of pyruvate $1^{-1}$ on oocyte maturation. The addition of glucose to medium containing $1 \mathrm{mmol}$ pyruvate $1^{-1}$ prevented the stimulatory effects of the higher concentration of pyruvate on oocyte maturation, as shown by Downs and Mastropolo (1994) and Downs (1995), as well as the increased consumption of pyruvate. These results raise two important possibilities regarding the actions of energy substrates on the oocyte: (1) that pyruvate and glucose may each influence oocyte maturation, at least in part, by modulating the consumption of the complementary energy substrate; and (2) that increased pyruvate consumption by the oocyte-cumulus cell complex drives the induction of oocyte maturation.

The first possibility was addressed by analysing oocyte maturation and substrate consumption or production in microdrop cultures using medium supplemented with hypoxanthine containing different combinations of pyruvate and glucose. Consistent with earlier experiments, a high concentration of pyruvate in the absence of glucose yielded a high frequency of maturation, and this was associated with a greater consumption of the substrate. The greatest percentage of maturation was observed in the group cultured in $5.5 \mathrm{mmol}$ glucose $\mathrm{l}^{-1}$ alone. Consumption of either pyruvate or glucose was suppressed by the addition of the complementary energy substrate, and this was mirrored in each instance by a drop in the maturation frequency. Glucose consumption in the group containing glucose alone was $2.06 \mathrm{nmol}$ per complex but this was reduced to 1.07 and $0.48 \mathrm{nmol}$ per complex, respectively, upon addition of 0.23 and $1 \mathrm{mmol}$ pyruvate $l^{-1}$. By the same token, pyruvate consumption was reduced upon the addition of glucose: adding glucose to $1 \mathrm{mmol}$ pyruvate $1^{-1}$ reduced pyruvate consumption by over $50 \%$; and adding glucose to $1 \mathrm{mmol}$ pyruvate $\mathrm{l}^{-1}$ led to a net accumulation of pyruvate, indicating that pyruvate production from glycolysis exceeded its consumption by the complex. The negative regulation of pyruvate or glucose utilization by the other substrate could occur through the action of reciprocal Crabtree and Pasteur effects, and increases in glycolytic or TCA cycle activity could limit the activity of the complementary pathway (Koobs, 1972). This may also explain the meiosis-blocking effect of either substrate when added to medium containing inhibitor supplemented with millimolar concentrations of the other substrate.

The energy substrate dynamics exhibited in the experiment just discussed are inconsistent with the idea that pyruvate consumption is a primary component of the meiotic induction mechanism. If increasing the pyruvate concentration is expected to augment GVBD, then the addition of pyruvate to medium containing $5.5 \mathrm{mmol}$ glucose $\mathrm{l}^{-1}$ should increase the percentage of maturation. Instead, the maturation frequency declined from $87 \%$ to $49 \%$ and $37 \%$, respectively, upon the addition of 0.23 and 1 mmol pyruvate $l^{-1}$. We have reported a similar finding in mouse oocytes cultured in Waymouth's MB $752 / 1$ medium, which contains a very high concentration $\left(27.8 \mathrm{mmol} \mathrm{l}^{-1}\right)$ of glucose (Downs and Mastropolo, in press). Moreover, as discussed above, net pyruvate consumption was observed in medium containing $1 \mathrm{mmol}$ pyruvate $\mathrm{I}^{-1}$ plus glucose, a condition that yielded a low percentage of oocyte maturation; on the other hand, pyruvate accumulated in medium containing only glucose, when a high percentage of maturation was observed. 
The high frequency of oocyte maturation observed in the group cultured in glucose alone was unexpected in the light of previous experiments that showed little effect of this hexose alone at comparable concentrations in medium containing inhibitor in the absence of FSH (Downs and Mastropolo, 1994). The subsequent experiment in this study comparing oocyte maturation in microdrops versus tubes demonstrated a dosedependent stimulatory effect of glucose alone in microdrops but not in tubes. This meiotic stimulation by glucose was consistent with the results of the previous experiment, although the magnitude of the response to glucose was not as great. The stimulatory action of glucose in microdrops but not tubes is puzzling, since if glucose is stimulatory, one would expect the effect in tubes to at least equal, or perhaps exceed, that observed in microdrops, due to a greater depletion of glucose in the microdrops. One possible explanation for this discrepancy is that depletion of some other medium component occurs in microdrops but not in tubes that allows the glucose to become stimulatory to oocyte maturation.

Additional experiments were carried out to investigate the association between pyruvate consumption and the resumption of meiotic maturation. When energy substrate concentrations were determined in cultures of spontaneously maturing cumulus cell-enclosed oocytes, a very different metabolic profile was apparent during the first $3 \mathrm{~h}$ of culture compared with the second $3 \mathrm{~h}$. During the first $3 \mathrm{~h}$, no net change in pyruvate was observed; glucose was consumed and an equivalent amount of lactate produced. However, a sharp reduction in glucose consumption was observed during the second $3 \mathrm{~h}$ period, while at the same time a significant amount of pyruvate was now consumed. Because $>93 \%$ of the oocytes had undergone GVBD after $3 \mathrm{~h}$ of culture, these data suggest that a shift to increased pyruvate consumption coincides with meiotic resumption and could conceivably serve as a metabolic marker for this meiotic event. Consistent with this idea was an earlier finding that pyruvate ceased to accumulate in culture medium containing FSH-treated, oocyte-cumulus cell complexes at about the time meiotic resumption was initiated (Downs et al., 1996). Indeed, oxygen consumption increases after GVBD in rat oocytes, when maturation has occurred either spontaneously or in response to hormone treatment (Magnusson et al., 1977; Ahren et al., 1978). This increase is blocked when meiotic arrest is maintained with dibutyryl cAMP, and it has been suggested that meiotic resumption is a trigger for increased oocyte respiration (Ahren et al., 1978). An exception may be the rabbit oocyte in which an increase in oxygen metabolism precedes nuclear maturation (Magnusson et al., 1981). However, the change in pyruvate utilization by mouse complexes cannot be permanent, because when the spent medium from spontaneously maturing mouse oocytes was assayed after $18 \mathrm{~h}$ of culture, net pyruvate production was observed ( $0.051 \mathrm{nmol}$ per complex). A more detailed kinetic analysis will be required to determine the extent of metabolic shifts throughout meiosis.

When oocyte-cumulus cell complexes were treated with DCA, an activator of pyruvate dehydrogenase (Whitehouse et al., 1974), consumption of pyruvate was increased but no effect on oocyte maturation was observed. It is presumed that the increase in pyruvate utilization was due to conversion of pyruvate to acetyl $\mathrm{CoA}$, which could then feed into the TCA cycle. The results with DCA demonstrate that augmenting pyruvate consumption does not necessarily lead to oocyte maturation and do not support the contention that oxidation of pyruvate is the mechanism by which high pyruvate concentrations lead to GVBD.

In the presence of a suitable energy source, oxygen has been shown to promote the maturation of denuded mouse and rat oocytes (Zeilmaker et al., 1972; Zeilmaker and Verhamme, 1974). Although oxygen consumption is apparently required by the oocyte for GVBD, it may not be a component of the induction process and may simply serve a permissive role in meiotic resumption. In support of this idea, lower concentrations of pyruvate $\left(0.23 \mathrm{mmol} \mathrm{I}^{-1}\right)$ support oocyte maturation, yet higher concentrations $\left(1 \mathrm{mmol} \mathrm{^{-1 }}\right)$ can induce maturation in the absence of any significant effect on oxygen consumption. This finding contrasts with studies in rats in which pyruvate stimulates respiration in both complexes and denuded oocytes (Hillensjo et al., 1975). Also, the addition of glucose to medium containing $1 \mathrm{mmol}$ pyruvate $1^{-1}$ reduced pyruvate consumption and maturation frequency, yet had no significant effect on oxygen consumption. Moreover, no effect of pyruvate on the ATP concentration in complexes was observed during a $3 \mathrm{~h}$ culture period, while glucose produced a $24-28 \%$ increase (Downs and Mastropolo, 1994). Finally, high oxygen tension was shown to be detrimental to the maturation of mouse (Haidri et al., 1971), hamster (Gwatkin and Haidri, 1973) and pig (Tsafriri and Channing, 1975) oocytes.

When mouse cumulus cell-enclosed oocytes are maintained in meiotic arrest with purines or CAMP analogues, FSHinduced GVBD depends on the presence of glucose (Fagbohun and Downs, 1992; Downs and Mastropolo, 1994) but it is not known whether pyruvate utilization is also required. Two different experiments were carried out to examine the effect of FSH on pyruvate consumption and oocyte maturation. In the first experiment, complexes were cultured in glucose-free medium containing hypoxanthine, and pyruvate consumption was measured after varying the duration of culture in the presence or absence of FSH. In the second experiment, complexes were cultured for $17-18 \mathrm{~h}$ in glucose-free medium containing either 0.23 or $0.46 \mathrm{mmol}$ pyruvate $\mathrm{I}^{-1}$, in the presence or absence of FSH. Both of these experiments failed to demonstrate a stimulatory effect of FSH on pyruvate consumption by complexes. Furthermore, although twice as much pyruvate was utilized at the higher pyruvate concentration, no significant difference in GVBD was observed despite the presence of FSH. Thus, not only did FSH fail to stimulate pyruvate consumption but it did not stimulate oocyte maturation when a greater amount of pyruvate was utilized by the complex. Therefore, it can be concluded that increased pyruvate utilization in the absence of glucose is not sufficient to bring about FSH-induced meiotic maturation. This is inconsistent with a requirement for pyruvate oxidation in the meiotic induction process.

Additional support for this idea came from the finding that FSH suppresses oxygen consumption by complexes in culture medium containing hypoxanthine and glucose at all the time points tested, up until the time meiotic induction was initiated. This is in agreement with previous studies in rats that showed a decrease in oxygen consumption by oocyte-cumulus cell complexes in response to gonadotrophin treatment in vivo or in 
vitro before meiotic resumption (Dekel et al., 1976; Magnusson et al., 1977; Ahren et al., 1978; Billig and Magnusson, 1985). Such a decrease has been attributed to a Crabtree effect due to augmented glycolytic activity in the cumulus cells (Dekel et al., 1976; Magnusson et al., 1977). The fact that depressed oxygen consumption occurs before GVBD in mice provides further evidence that pyruvate oxidation does not serve a mediating role in hormone-induced maturation, and, in fact, suggests the contrary. It is well established that glucose is required for meiotic induction (Fagbohun and Downs, 1992; Downs and Mastropolo, 1994) but glucose is not acting in this capacity by supplying pyruvate as a glycolytic end-product for consumption by the oocyte, because the addition of glucose to pyruvate-containing medium actually reduces pyruvate consumption or even results in pyruvate accumulation.

In conclusion, while it is clear that pyruvate can have a positive influence on oocyte maturation, its actions on meiotic induction do not appear to function primarily via metabolism through the TCA cycle. Pyruvate may act by means other than as an energy source, for example, it may modulate protein or lipid synthesis, or function as an antioxidant (as has been suggested by Andrae et al., 1985; O'Donnell-Tormey et al., 1987; and Leese, 1990). Elucidation of the mechanism(s) by which carbohydrates such as pyruvate and glucose influence meiotic maturation will constitute an important advance in our understanding of meiotic regulation in mammals.

This research was supported by grants from the NIH (HD25291 and TWO2079) to S. M. Downs. Special thanks to A. Mastropolo for technical assistance.

\section{References}

Andrae U, Singh J and Ziegler-Skylakakis K (1985) Pyruvate and related $\alpha$-ketoacids protect mammalian cells in culture against hydrogen peroxideinduced cytotoxicity Toxicology Letters $2893-98$

Ahren K, Dekel N, Hamberger L, Hillensjo T, Hultborn R, Magnusson C and Tsafriri A (1978) Metabolic and morphological changes produced by gonadotrophins and cyclic AMP in the oocyte and cumulus oophorus of preovulatory rat follicles Annales de Biologie animale Biochemistry and Biophysics 18 409-417

Biggers JK, Whittingham DG and Donahue RP (1967) The pattern of energy metabolism in the mouse oocyte and zygote Proceedings of the National Academy of Sciences USA 58 560-567

Billig H and Magnusson C (1985) Gonadotropin-induced inhibition of oxygen consumption in rat oocyte-cumulus complexes: relief by adenosine Biology of Reproduction 33 890-898

Dekel N and Beers WH (1978) Rat oocyte maturation in vitro: relief of cyclic AMP inhibition by gonadotropins Proceedings of the National Academy of Sciences USA 75 4369-4373

Dekel N, Hultborn R, Hillensjo T, Hamberger L and Kraicer P (1976) Effect of luteinizing hormone on respiration of the preovulatory cumulus oophorus of the rat Endocrinology 98 498-504

Donahue RP and Stern S (1968) Follicular cell support of oocyte maturation: Production of pyruvate in vitro. Journal of Reproduction and Fertility 17 395-398

Downs SM (1995) The influence of glucose, cumulus cells, and metabolic coupling on ATP levels and meiotic control in the isolated mouse oocyte Developmental Biology 167 502-512
Downs SM and Mastropolo AM (1994) The participation of energy substrates in the control of meiotic maturation in murine oocytes Developmental Biology 162 154-168

Downs SM and Mastropolo AM Culture conditions affect meiotic regulation in cumulus cell-enclosed mouse oocytes Molecular Reproduction and Development (in press)

Downs SM, Daniel SAJ and Eppig JJ (1988) Induction of maturation in cumulus cell-enclosed mouse oocytes by follicle-stimulating hormone and epidermal growth factor: evidence for a positive stimulus of somatic cell origin Journal of Experimental Zoology 245 86-96

Downs SM, Humpherson PG, Martin KL and Leese HJ (1996) Glucose utilization during gonadotropin-induced meiotic maturation in cumulus cell-enclosed mouse oocytes Molecular Reproduction and Development 44 121-131

Edwards RG (1965) Maturation in vitro of mouse, sheep, cow, pig, rhesus monkey and human ovarian oocytes Nature 208 349-351

Eppig JJ (1976) Analysis of mouse oogenesis in vitro: oocyte isolation and the utilization of exogenous energy sources by growing oocytes Journal of Experimental Zoology 198 375-382

Eppig J and Downs SM (1984) Chemical signals that regulate mammalian oocyte maturation Biology of Reproduction 30 1-11

Fagbohun CF and Downs SM (1992) Requirement for glucose in ligandstimulated meiotic maturation of cumulus cell-enclosed mouse oocytes Journal of Reproduction and Fertility 96 681-697

Gwatkin RBL and Haidri AA (1973) Requirements for the maturation of hamster oocytes in vitro. Experimental Cell Research 76 1-7

Haidri AA, Miller IM and Gwatkin RBL (1971) Culture of mouse oocytes in vitro, using a system without oil or protein Journal of Reproduction and Fertility 26 409-411

Hillensjo T, Hamberger L and Ahren K (1975) Respiratory activity of oocytes isolated from ovarian follicles of the rat Acta Endocrinologica 78 751-759

Houghton FD, Thompson JG, Kennedy CJ and Leese HJ (1996) Oxygen consumption and energy metabolism of the early mouse embryo Molecular Reproduction and Development 44 476-485

Koobs DH (1972) Phosphate mediation of the Crabtree and Pasteur effects Science 178 127-133

Leese HJ (1990) Physiology of the fallopian tube: the provision of nutrients for oocytes and early embryos. In Ovulation to Implantation pp 121-125 Eds JHL Evers and MJ Heineman. Elsevier Science Publishers, New York

Leese HJ and Barton AM (1985) Production of pyruvate by isolated mouse cumulus cells Journal of Experimental Zoology 234 231-236

Magnusson C, Hillensjo T, Tsafriri A, Hultborn R and Ahren K (1977) Oxygen consumption of maturing rat oocytes Biology of Reproduction 17 9-15

Magnusson C, LeMaire WJ and Hillensjo T (1981) Stimulation by hCG in vivo of oxygen consumption by rabbit oocytes in vitro. Journal of Reproduction and Fertility 61 185-188

O'Donnell-Tormey J, Nathan CF, Lanks K, DeBoer CJ and De La Harpe J (1987) Secretion of pyruvate: an antioxidant defence of mammalian cells Journal of Experimental Medicine 165 500-514

Pincus G and Enzmann EV (1935) The comparative behavior of mammalian eggs in vivo and in vitro. I. The activation of ovarian eggs Journal of Experimental Medicine 62 665-675

Schultz RM (1991) Meiotic maturation of mammalian oocytes. In Elements of Mammalian Fertilization pp 77-104 Ed. PM Wassarman. CRC Press, Boston

Tsafriri A and Channing CP (1975) Influence of follicular maturation and culture conditions on the meiosis of pig oocytes in vitro. Journal of Reproduction and Fertility 43 149-152

Whitehouse S, Cooper RH and Randle PJ (1974) Mechanism of activation of pyruvate dehydrogenase by dichloroacetate and other halogenated carboxylic acids Biochemical Journal 141 761-774

Zeilmaker GH and Verhamme CMPM (1974) Observations on rat oocyte maturation in vitro: morphology and energy requirements Biology of Reproduction 11 145-152

Zeilmaker GH, Hulsmann WC, Wensinck F and Verhamme C (1972) Oxygentriggered mouse oocyte maturation in vitro and lactate utilization by mouse oocytes and zygotes Journal of Reproduction and Fertility 29 151-152 